\title{
Saber y objetivación. Reflexiones en torno a la caja de herramientas foucaulteana
}

\author{
Dr. Iván Gabriel Dalmau'
}

Recibido: 5 de diciembre de 2018 - Aceptado: 13 de marzo de 2019

\begin{abstract}
Resumen
En el presente artículo nos proponemos llevar a cabo una relectura del abordaje foucaulteano del saber, desde la perspectiva del modo en que el filósofo problematizó el carácter crítico de su actividad filosófica. En ese sentido, apoyados en el característico gesto de recuperación y reelaboración recurrente que caracteriza el modo de trabajo de Michel Foucault, revisaremos el archivo foucaulteano con el objetivo de constituir una serie documental que nos permita articular las nociones de crítica y saber. Específicamente nos preguntamos, ¿qué tipo de crítica de las ciencias humanas, en tanto saberes, posibilita la puesta en juego de la caja de herramientas foucaulteana?
\end{abstract}

Palabras clave: Foucault - saber - objetivación - crítica - ciencias humanas

\section{Knowledge and objectivation. Reflections on the Foucault's toolbox}

\begin{abstract}
This article proposes a rereading of Foucault's treatment of knowledge, from the perspective of the way in which the philosopher problematized the critical nature of his philosophical activity. In this sense, and supported by the characteristic gesture of recovery and recurring recreation in the work of Michel Foucault, we will look at Foucault's archives in order to
\end{abstract}

1 Doctor en Ciencias Sociales (UBA, Argentina). Becario Posdoctoral del Consejo Nacional de Investigaciones Científicas y Técnicas (CONICET, Argentina), radicado en la Escuela de Humanidades de la Universidad Nacional de San Martín (UNSAM, Argentina). Docente de Epistemología de las Ciencias Sociales (Humanidades - UNSAM) y de Introducción al Pensamiento Científico en el Ciclo Básico Común de la Universidad de Buenos Aires (UBA, Argentina). Ha realizado estancias de investigación como invitado en la Universidad Autónoma de Madrid (UAM, Estado Español) y en la Universidad de Granada (Estado Español).

Contacto: ivandalmau@yahoo.com.ar

ORCID: https://orcid.org/0000-0003-4045-3488 
put together a series of documents in order to articulate the notions of critical thinking and knowledge. Specifically, we ask ourselves, what type of critical thinking in the human sciences, and other areas of knowledge, enables the application of Foucault's toolbox?

Key words: Foucault - knowledge-objectivation - critique - human sciences

\section{Saber e objetivação. Reflexões em torno da caixa de ferramentas foucaulteana}

\section{Resumo}

No presente artigo nos propomos realizar una releitura da abordagem foucaulteana do saber, desde a perspectiva do modo em que o filósofo problematizou o carácter crítico de sua atividade filosófica. Nesse sentido, apoiados no característico gesto de recuperação e reelaboração recorrente que caracteriza o modo de trabalho de Michel Foucault, revisaremos o arquivo foucaulteano com o objetivo de constituir uma série documental que nos permita articular as noções de crítica e saber. Especificamente nos perguntamos, que tipo de crítica das ciências humanas, quanto saberes, pode ativar a caixa de ferramentas foucaulteana?

Palavras-chave: Foucault - saber - objetivação - crítica - ciências humanas

\section{A modo de introducción ${ }^{2}$}

"No se trata ni de exponer lo que los científicos o los sabios han dicho o pensado o creído demostrar ni de reconstruir desde un punto de vista verdadero o de la ciencia normal, desde el punto de vista contemporáneo, lo que se ha dicho o pensado o demostrado, considerando el presente de una ciencia como normativo respecto de su pasado. (...) La arqueología del saber procura establecer a partir de qué disposición histórica han sido posibles las ciencias, sin identificar

tal disposición ni con el valor racional del conocimiento ni con sus formas objetivas" (Castro 1995 39).

En el presente escrito se pretende revisar la problematización foucaulteana del saber, de modo tal de rastrear herramientas que permitan

2 Quisiera agradecer especialmente al Dr. Claudio Cormick y al Lic. Mario Gómez Pedrido por las críticas dirigidas a versiones previas de este trabajo. 
calibrar el ejercicio de la crítica de las ciencias humanas. Sería pertinente destacar que no se trata de llevar a cabo una reconstrucción de las múltiples aproximaciones que pueden encontrarse en los trabajos rubricados por Michel Foucault respecto de dichas ciencias, sino más bien de dar cuenta de la cantera de trabajo filosófico que se abre al problematizarlas en tanto saberes. En sintonía con la cita del reconocido investigador Edgardo Castro que hemos colocado como epígrafe, tomamos como punto de partida que la arqueología foucaulteana no se ocupa de llevar a cabo una epistemología normativa de las ciencias humanas ${ }^{3}$, y nos preguntamos entonces: ¿qué tipo de crítica permite calibrar el abordaje arqueológico?

Ahora bien, para dar cuenta del interrogante explicitado, constituiremos una serie documental que nos permita captar la recurrencia temática del abordaje de las ciencias humanas ${ }^{4}$ en los trabajos foucaulteanos y su estrecha ligazón con la problematización de la filosofía como una actividad de diagnóstico de la actualidad. De este modo, apoyados en el característico gesto foucaulteano de recuperación y reelaboración recurrente de sus trabajos precedentes (Fontana y Bertani 1997 248), que se ha tornado insoslayable tras la edición en formato libro de sus cursos dictados en el Collège de France (Raffin 2015 58-63), estaremos en condiciones de revisar la arqueología foucaulteana de los saberes acerca de "lo humano" desde la perspectiva del modo en que el filósofo problematiza la crítica filosófica.

En lo que respecta a la organización formal, cabe destacar que dividiremos las líneas que se despliegan a continuación en tres parágrafos y un breve apartado de reflexión final. En el primero explicitaremos

\footnotetext{
3 Si bien citamos al reconocido especialista argentino Edgardo Castro, cabe destacar que el reconocimiento de que el abordaje foucaulteano del saber no se encuentra motorizado por una preocupación de índole normativa es una posición mayoritariamente compartida por los especialistas, de quienes nos haremos eco a lo largo del artículo (Gutting 1989 227-260; Han 1998 66-118; Sauquillo 2001 17-23; Sabot 2006 1-33; Giordano 2007 42-72; Raffin 2008; Mascaretti 2014; Paltrinieri 2014).

4 Nótese que nos ocuparemos de revisar la recurrencia temática en pos del objetivo del artículo, mas no de reconstruir la lectura elaborada por el filósofo en cada una de sus aproximaciones, cual si fuera otra la pregunta que animara la escritura de nuestro trabajo.
} 
someramente la estrategia de lecto-escritura filosófica implementada en nuestro trabajo sobre el "archivo" Foucault. Luego nos detendremos en el abordaje recurrente de las ciencias humanas en los trabajos foucaulteanos, enmarcándolos en la manera en que buscó ligar su producción filosófica al diagnóstico de la actualidad. Tras lo cual, apoyados en dicha revisión, desplegaremos una relectura de la arqueología foucaulteana del saber, tal como el filósofo la problematizara en su clásico libro de finales de los años 60 (Foucault 1969), en el que desarrolla una revisión y reelaboración crítica de las herramientas puestas en juego en sus indagaciones precedentes (Nosetto 2010 73-76; Beresñak 2014 39-43).

\section{Punto de partida: breves reflexiones acerca de la estrategia de lecto-escritura filosófica}

Antes de dar paso a los parágrafos en los que se abordan los aspectos sustantivos del presente artículo, consideramos fundamental interpolar estas líneas de reflexión acerca de la metodología de lecto-escritura filosófica implementada. En primer lugar, querríamos remarcar que esta breve problematización acerca del método de trabajo filosófico parte de una apropiación de las herramientas forjadas por Michel Foucault. Básicamente, a partir de nuestra lectura de la caja de herramientas foucaulteana, consideramos pertinente realizar la siguiente "distinción categorial": el preguntarnos respecto de las herramientas constituidas por la filosofía foucaulteana en absoluto implica comprometernos con un análisis doxológico respecto de lo ajustado o no de las referencias introducidas por Foucault respecto de otras/os filósofas/os y corrientes filosóficas. Por lo tanto, al revisar el modo en que problematiza el saber, distinguiéndolo del conocimiento, no pretendemos evaluar la prolijidad de las referencias foucaulteanas a la fenomenología, por ejemplo; como así tampoco nos preocuparemos por la "corrección" de sus lecturas de Immanuel Kant o Friedrich Nietzsche.

A partir de la "distinción categorial" propuesta, se dejan de lado un conjunto de formas de problematización: al revisar las herramientas constituidas por el discurso foucaulteano, nos colocaremos por fuera de la dicotomía formada por la asunción acrítica de sus referencias 
a otras corrientes filosóficas, como del otro extremo, formado por la "crítica por elevación" que, desconociendo la distinción propuesta, pretendería pasar, sin solución de continuidad, de la puesta en entredicho de la lectura foucaulteana de la fenomenología al cuestionamiento de las herramientas configuradas por Foucault en tanto contracara de sus referencias a dicha corriente. Distinguiremos, por lo tanto, entre el modo en que las referencias a otras corrientes filosóficas estructuran el discurso foucaulteano, articulando su economía, y la evaluación respecto de la plausibilidad filológico-historiográfica de las mismas.

Revisaremos entonces el discurso foucaulteano de manera análoga a la que el propio Foucault implementara en su abordaje de los trabajos configurados a partir de mediados del siglo XVI, que tenían a la crítica de Maquiavelo como punto de partida. Trabajos acerca de los cuales el filósofo francés explicitó que no se preocuparía respecto de lo ajustado o no de la lectura que se hacía de los textos del clásico pensador político florentino, sino del modo en que en dichos discursos se ponía en juego la contraposición entre los "consejos del príncipe" y el ejercicio del gobierno, que era justamente el problema cuya constitución pretendía abordar (Foucault 2004a).

\section{El abordaje recurrente de las ciencias humanas dentro del marco del ejercicio de la crítica como diagnóstico de la actualidad}

"En retrospectiva, los trabajos publicados aparecen como fotografías, como recortes momentáneos de un proceso (...). Sin embargo, la lectura de las lecciones del Collège de France provee un antídoto eficiente para esto. En dichas conferencias vemos a Foucault trabajando, volviendo constantemente sobre cuestiones previas, retomándolas y reformulándolas (...)" (Wallenstein 2013 10).

En la primera clase del curso dictado en el Collège de France, durante el ciclo lectivo 1982-1983, del 5 de enero de 1983, con la que se diera inicio al curso Le gouvernement de soi et des autres, Foucault se vale de una presentación del modo en que Immanuel Kant responde 
a la pregunta "Was ist Aufklärung?", para inscribir su propia labor en una modulación de la crítica. Pregunta kantiana en la que el filósofo se detuvo de manera recurrente entre 1978 y 1984 (Foucault 1990 1994c); sin embargo, cabe destacar que la reconstrucción y el análisis respecto de las continuidades, rupturas y matices que atraviesan dichas aproximaciones, escapan de los objetivos y posibilidades de nuestro trabajo. Volviendo sobre la lección en cuestión, consideramos sugerente la contraposición esbozada en el siguiente fragmento:

Me parece que la elección filosófica a la que nos encontramos confrontados actualmente es ésta. Hay que optar por una filosofía crítica que se presentará como una filosofía analítica de la verdad en general, o por un pensamiento crítico que tomará la forma de una ontología de nosotros mismos, de una ontología de la actualidad. Y es esta forma de filosofía la que, de Hegel a la Escuela de Frankfurt, pasando por Nietzsche, Max Weber, etc., ha fundado una forma de reflexión a la cual, desde luego, me vinculo en la medida en que puedo (Foucault 2008 22).

Consideramos fundamental detenernos en que, frente a la realización de una "analítica de la verdad en general", preocupada por las posibilidades del conocimiento y sus límites infranqueables, Foucault reivindica la práctica de la crítica como una ontología de la actualidad, como una ontología de nosotros mismos. De lo que se trata entonces es de una inflexión del gesto crítico que, desanclado de todo viso de universalidad, se desliga a su vez de una pretensión fundadoranormativa. En lugar de configurarse como una gnoseología que dé cuenta de las condiciones de posibilidad del conocimiento, normando las formas adecuadas de conocer, y una ética orientada por principios que permitan normar moralmente la acción, la crítica foucaulteana opera por medio del cuestionamiento del pretendido carácter necesario de las formas de pensamiento y de los modos de conducción de los sujetos en sus relaciones consigo mismos y con los otros (Gros 2008 348-361). Cabe recordar que dicha preocupación ontológicopolítica respecto de la actualidad se liga en la perspectiva foucaulteana a la problematización de focos de experiencia, constituidos a partir 
de la imbricación entre las formas de saber, las matrices normativas de comportamiento y los modos de existencia virtual para sujetos posibles. Al respecto, en el marco de su revisión del proyecto crítico foucaulteano, el investigador Giovanni Maria Mascaretti sostuvo que:

(...) El objetivo de las investigaciones arqueológico-genealógicas de Foucault no es solamente describir problematizaciones históricas, sino además desenmascararlas y desafiarlas por medio del cuestionamiento de la inevitabilidad y necesidad racional de prácticas, instituciones, técnicas y funciones que han sido construidas como respuestas suyas (Mascaretti 2014 139).

Ahora bien, nuestra estrategia de relectura de la problematización arqueológica del saber, elaborada por Foucault desde la perspectiva crítica de su trabajo filosófico, requiere, para quedar al abrigo de una objeción que marcara el presunto carácter teleológico de la propuesta, de la realización de una serie de movimientos. Así, no podemos ceñirnos exclusivamente a revisar las nociones de "crítica" y "saber" en el archivo Foucault, sino que además debemos reponer una serie documental complementaria que nos permita sostener la relectura realizada. Por lo tanto, se nos impone la tarea de revisar, al menos someramente, los problemas que jalonan las indagaciones arqueológicas respecto de las ciencias humanas desplegadas por Foucault en los años sesenta, de modo de señalar las preocupaciones ontológicopolíticas ligadas al diagnóstico de la actualidad que se encontraban a la base de dichas problematizaciones. Vía de abordaje del archivo que consideramos que debe completarse con el señalamiento de lo que podríamos denominar como "ecos arqueológicos de la genealogía", en sintonía con la cita de Gary Gutting que introducimos a continuación:

La arqueología (...) continúa jugando un rol clave en los trabajos posteriores de Foucault. Por consiguiente, la reflexión respecto del abordaje de la práctica moderna de castigo de los criminales por medio de la prisión, realizada en Vigilar y Castigar, revela que ésta involucra los cuatro aspectos fundamentales de una formación discursiva. (...) 
Esta aplicación de los análisis arqueológicos no es una innovación, sino más bien un retorno a las aproximaciones de Historia de la locura, en donde, por ejemplo, la arqueología mostró la estructura común del discurso filosófico cartesiano y la práctica Clásica no-discursiva del encierro y reveló la relevancia moral del asilo del siglo diecinueve (Gutting 1989 270-271).

Antes de proseguir nuestra lectura, se nos impone la necesidad de introducir la siguiente aclaración terminológica. Si bien por "ciencias humanas" Foucault alude a los estudios literarios, la sociología y las ciencias con radical "psi", en la medida en que remarca que las mismas se configuraron a partir del plexo formado por las ciencias respecto de la vida, el trabajo y el lenguaje, para simplificar la redacción nos valemos de la noción de "ciencias humanas" en sentido amplio y englobante, para aludir a la disposición epistémica en la que dichas ciencias se formaron (Foucault 1966). Del mismo modo, tanto respecto de la arqueología de la constitución del objeto locura como "enfermedad mental" (Foucault 1972)5, como de la relativa al nacimiento de la mirada médica (Foucault 1963), puede plantearse que se engarzan con el problema de la constitución del "hombre", en tanto objeto de saber que vive, trabaja y habla. Por lo tanto, incluimos dichas investigaciones dentro de la interrogación foucaulteana acerca de la formación de saberes respecto de "lo humano"; vinculación que, en el caso de la arqueología de la medicina clínica, fuera destacada por el especialista Philippe Sabot: "las últimas páginas de Naissance de la clinique destacaban fuertemente la correlación arqueológica entre la emergencia del concepto moderno de vida y el nacimiento de las ciencias humanas" (Sabot 2006 60).

Sin embargo, el señalamiento de dicha recurrencia problemática y del mencionado solapamiento temático, bajo ningún punto de vista implica asumir un compromiso historiográfico respecto de una supuesta

5 Para evitar confusiones, remarcamos que hemos trabajado sobre la segunda edición francesa -publicada en 1972-, pero que la versión original de dicha investigación, presentada por el filósofo como tesis doctoral, es de 1961. 
"síntesis superadora" de las mencionadas indagaciones "fragmentarias". Por un lado, nada se encuentra más alejado de la estrategia que reivindicamos como método de lecto-escritura filosófica; por otro, no puede desconocerse que, por medio de la problematización de las formaciones discursivas como herramientas de trabajo filosófico -a las que aludiremos en el próximo parágrafo-, Foucault busca tomar distancia de las lecturas "totalizantes" en términos de "paradigma" a las que habilitaba la noción de episteme, tal cual fue puesta en juego en Les mots et les choses (Foucault 1966).

Consideramos, entonces, que no puede pasarse por alto que en L'archéologie du savoir (Foucault 1969), el filósofo se ocupa de manera recurrente de revisar y reformular parcialmente su abordaje de los saberes desplegado en sus arqueologías precedentes, respecto de "la locura", "la clínica" y "el hombre". Si en los dos primeros trabajos la preocupación por la imbricación entre la formación de determinados saberes y la transformación de las prácticas institucionales -de segregación y encierro, por ejemplo- resulta ineludible, en su arqueología del "hombre" Foucault parecería circunscribir el trabajo de archivo exclusivamente a los discursos científicos, resultando en principio menos palpable la articulación entre dicha indagación y una preocupación ontológico-política que permita ligar esta pesquisa a las tareas de diagnóstico de la actualidad.

Sin embargo, cabría recordar que la apuesta foucaulteana radica en llevar a cabo una problematización que le permita escapar de las mallas del humanismo decimonónico, es decir, que en su arqueología respecto de la constitución del "hombre", la búsqueda foucaulteana radica justamente en dar cuenta de las condiciones de posibilidad de la formación de dicha figura epistemológica, de modo tal de poder desasirse de la grilla de inteligibilidad que hace del "hombre" el punto

6 Para una lectura centrada en dicho "clásico" foucaulteano, resultan ineludibles los tempranos trabajos de Gary Gutting (1989) y Edgardo Castro (1995). Por otra parte, en lo que respecta a la realización de una reconstrucción minuciosa de las múltiples declinaciones de la noción de "arqueología" en Foucault, no podemos dejar de mencionar el trabajo de Béatrice Han (1998 7-28). 
de partida tanto de la reflexión teórico-epistemológica como de la teorización práctico-política. En este contexto, consideramos más que atinada la interpretación propuesta por Luca Paltrinieri, quien respecto de la vinculación entre la indagación arqueológica y la preocupación por el diagnóstico crítico de la actualidad sostuvo que: "si la historia posee un privilegio en la óptica foucaulteana, es precisamente porque permite abstraerse del propio presente, tomar distancia de la propia cultura cotidiana para examinar 'desde el exterior' nuestro sistema de certezas y discursos" (Paltrinieri 2014 108). En lo que a la figura del "hombre" respecta, no puede soslayarse la elocuente pero repudiable metáfora de la que Foucault se vale en 1967 para desmarcarse de la generación filosófica que lo antecedió, al caracterizar el humanismo como "la prostituta" de todo el pensamiento, la cultura, la moral y la política de las décadas precedentes (Foucault 1994d).

Dejando a un lado la discusión propia de la "historia de las ideas" (Foucault 1969 9-28) en torno de la pertenencia o no de Foucault a la "tradición" estructuralista, no puede desconocerse que en Les mots et les choses (Foucault 1966), tras reconstruir la configuración de la episteme moderna, frente a las ciencias humanas Foucault caracteriza el psicoanálisis y la etnología como "contraciencias". Además, en el contexto de la profusa recepción de dicho libro, en el marco de una entrevista que le hicieran en 1967 el filósofo sostuvo que "la filosofía estructuralista permite diagnosticar qué es la actualidad" (Foucault 1994e), y de 1968 son tanto su polémica con Sartre (Foucault 1994f) como su respuesta a una carta de lectores en la que se detiene en la posibilidad de pensar "una política progresista no humanista" (Foucault 1994g). En ese sentido, consideramos que no puede pasarse por alto el modo en que Foucault se remite a Nietzsche en 1966 para, en el contexto de responder a la pregunta "qué es un filósofo", caracterizar el tipo de labor filosófica desplegada por medio de sus trabajos arqueológicos:

(...) A propósito de Nietzsche, podemos volver sobre su pregunta: para él, el filósofo es quien diagnostica el estado del pensamiento. Por cierto, se pueden concebir dos clases de filósofos, el que abre nuevos caminos al pensamiento, 
como Heidegger, y el que juega en cierta forma el rol de arqueólogo, que estudia el espacio en el que se despliega el pensamiento, así como sus condiciones, su modo de constitución (Foucault 1994h 553).

Por otra parte, cabe recordar que hacia el final de L'archéologie du savoir, Foucault se explaya sobre la posibilidad de llevar a cabo "otras arqueologías", entre las que destacan, además de la pintura, la sexualidad y la política, en sintonía con los blancos de problematización en los que se detendría en los años subsiguientes (Castro 2011a 43-45), en el marco tanto de sus libros publicados en vida como de los cursos dictados en el Collège de France. Siguiendo la lectura propuesta por Daniel Defert: "la arqueología como método, y específicamente Las Palabras y las Cosas, son una propedéutica a la genealogía (...) la genealogía, tal como nos fue presentada por Foucault, no es la crisis de la arqueología, sino que ellas se apuntalan mutuamente" (Defert 2011 271), sostenemos que en los trabajos genealógicos se palpan "ecos arqueológicos" que podrían distinguirse en tres dimensiones. Por un lado, en el nivel de la propia palabra foucaulteana encontramos que, explícitamente, se propone retomar tanto la noción de "saber" como la de "prácticas discursivas" para abordar los sistemas de pensamiento. En el resumen correspondiente al curso dictado en el Collège de France durante el ciclo lectivo 1970-71, el filósofo explicitó que:

Investigaciones realizadas previamente permitieron reconocer un nivel singular entre aquellos que permiten analizar los sistemas de pensamiento: el de las prácticas discursivas. Se trata de una sistematicidad que no es de tipo lógico ni lingüístico. Las prácticas discursivas se caracterizan por el recorte de un campo de objetos, la definición de una perspectiva legítima para el sujeto de conocimiento, la fijación de normas para la elaboración de conceptos y teorías. Cada una de ellas supone, entonces, un juego de prescripciones que rigen las exclusiones y elecciones. (...) Los caracteres generales de esas prácticas y los métodos propios para analizarlas se inventariaron bajo el nombre de arqueología (Foucault 2011 217-218). 
En este contexto, cabría recordar que, en el marco de la Leçon sur Nietzsche, dictada en Montreal en 1971, el filósofo se enfocó en la posibilidad erigida a partir de la genealogía nietzscheana de: "(...) pensar el conocimiento como un proceso histórico previo a toda problemática de la verdad, y más fundamentalmente que en la relación sujeto-objeto. El conocimiento liberado de la relación sujeto-objeto, es el saber" (Foucault 2011 205). A su vez, las notas particulares que remarcaremos a continuación, cuando planteemos que la arqueología permite calibrar un registro de problematización que se coloca por fuera de los pares sujeto-objeto y teleología-normación, se encuentran estrechamente ligadas al modo en que el filósofo caracteriza la genealogía como método para el trabajo filosófico que se despliega en las canteras de la historia (Foucault 1994i 1994j). En torno a lo cual el especialista Marcelo Raffin sostuvo que:

Foucault se propone mostrar cómo las prácticas sociales pueden llegar a engendrar dominios de saber que no sólo hacen que aparezcan nuevos objetos, conceptos y técnicas, sino que hacen nacer, además, formas totalmente nuevas de sujetos y sujetos de conocimiento. En este sentido, Foucault afirma que el sujeto de conocimiento posee una historia, la relación del sujeto con el objeto, y, más claramente, la verdad misma tiene una historia (Raffin 2014 130).

Por último, como tercera dimensión se destaca el abordaje recurrente de la formación de las ciencias humanas, justamente en el marco de los problemas mencionados en torno de las "otras arqueologías". Por ejemplo, en su genealogía de la prisión, en la que se ocupa de dar cuenta del surgimiento del modo disciplinario de ejercicio del poder, remarcará, acerca de la práctica del examen que caracteriza a los dispositivos disciplinarios, que dicha forma de ejercicio del poder se encuentra imbricada con la formación de saberes acerca de "lo humano", como la criminología (Foucault 1975). Resulta más que elocuente el modo en que presenta el objetivo del libro: "(...) Objetivo de este libro: una historia correlativa del alma moderna y de un nuevo poder de juzgar; una genealogía del actual complejo científico-judicial en el que el poder de castigar toma sus apoyos, recibe sus justificaciones $y$ 
sus reglas, extiende sus efectos y enmascara su exorbitante singularidad" (Foucault 1975 27).

En la misma línea, puede remarcarse la relevancia de la formación de dichos saberes en el contexto de la genealogía foucaulteana de la sexualidad (Foucault 1976) y de las formas modernas de gubernamentalidad (Foucault 2004a 2004b). Sobre este punto, hacemos propia la lectura del investigador Santiago Castro-Gómez: "tal vez una de las partes más interesantes de Seguridad, Territorio, Población sea la relectura que hace Foucault de su libro, publicado 12 años antes, Las palabras y las cosas, al final de la clase del 25 de enero de 1978" (Castro-Gómez 2010 16). En dicha clase, Foucault reformula su lectura respecto de la formación de las ciencias de la vida, el trabajo y el lenguaje, en cuyo seno se configuraron las ciencias humanas, al dar cuenta de la imbricación entre la formación de dichas ciencias y la constitución de la población como objeto de saber y blanco de intervención política de las tecnologías de seguridad características de las formas de gobierno económico (Foucault 2004a).

\section{La arqueología del saber como crítica de las formas de objetivación inmanentes a la formación discursiva de las ciencias humanas}

"Foucault entiende por "saber" las delimitaciones y las relaciones entre: 1) aquello de lo cual se puede hablar en una práctica discursiva (el dominio de los objetos); 2) el espacio en el que el sujeto puede ubicarse para hablar de los objetos (posiciones subjetivas); 3) el campo de coordinación y de subordinación de los enunciados en el que los conceptos aparecen, se definen, se aplican y se transforman; 4) las posibilidades de utilización y de apropiación de los discursos" (Castro 2011b 363).

Ahora bien, retomando lo señalado en la presentación del presente escrito, desarrollaremos a continuación una relectura de determinados fragmentos del "archivo Foucault", de modo tal de, apoyándonos en el característico gesto de recuperación y reelaboración recurrente que atraviesa sus trabajos, revisar su problematización arqueológica 
del saber desde la perspectiva de la inflexión del gesto crítico en que ha buscado ubicar su producción filosófica. Consideramos pertinente detenernos, entonces, en la forma de problematización de los discursos que Foucault propone a fines de los años 60 en su clásico L'archéologie du savoir:

Pero de lo que aquí se trata, no es de neutralizar el discurso, de hacerlo el signo de otra cosa y de atravesar su espesor para alcanzar aquello que permanece silenciosamente más allá de él sino, al contrario, de mantenerlo en su consistencia, de hacerlo surgir en la complejidad que le es propia (...). Sustituir el tesoro enigmático de las "cosas" anteriores al discurso, por la formación regular de los objetos que no se perfilan más que en él. Definir esos objetos sin referencia al fondo de las cosas, sino en relación al conjunto de reglas que permiten formarlos como objetos de un discurso y constituyen así sus condiciones de aparición histórica (Foucault 1969 65).

En dicho marco, la problematización arqueológica de los discursos consistirá en abordarlos en tanto prácticas y se orientará a dar cuenta de sus condiciones de posibilidad; logrando así el establecimiento de los modos históricos de constitución de ciertas positividades, en lugar de tomarlas de antemano como evidencia y punto de partida. Como lo planteara el investigador Alain Brossat: "el archivo, en suma, es lo que torna manifiesta la manera en que los discursos coexisten en un campo determinado, permanecen y, luego, se borran" (Brossat 2013 2-3). En este contexto, resulta relevante la inclusión de la definición dada por Foucault respecto de las formaciones discursivas en el capítulo segundo de la primera parte de L'archéologie du savoir, en donde el pensador planteó que:

En el caso en que se pudiera describir, entre un cierto número de enunciados, un semejante sistema de dispersión, en el caso en que entre los objetos, los tipos de enunciación, los conceptos, las elecciones temáticas, se pudiera definir una regularidad (un orden, correlaciones, posiciones y funcio- 
namientos, transformaciones), se dirá, por convención, que se trata de una formación discursiva, evitando así palabras demasiado cargadas de condiciones y de consecuencias, inadecuadas por otra parte para designar una dispersión semejante, como "ciencia", o "ideología", o "teoría", o "dominio de objetividad". Se llamarán reglas de formación a las condiciones a las que están sometidos los elementos de esta repartición (objetos, modalidad de enunciación, conceptos, elecciones temáticas). Las reglas de formación son condiciones de existencia (pero también de coexistencia, de conservación, de modificación y de desaparición) en una repartición discursiva determinada (Foucault 1969 53).

A través de la sucesión de capítulos que estructura dicha parte del libro, titulada "Les régularités discursives", el filósofo desgajará cada aspecto que configura la citada definición, apoyándose en los discursos que había abordado en sus trabajos precedentes y reformulando el abordaje de los mismos desde la perspectiva del "pasaje en limpio" de la metodología arqueológica y sus supuestos epistemológicos. El pasaje de la problematización de los objetos y las modalidades enunciativas hacia sus reglas de formación resulta un aporte epistemológico fundamental, puesto que permite desanclar la epistemología de las ciencias humanas del interior de la relación sujeto-objeto y visibilizar los términos de la relación cognoscitiva como inmanentes a dichas reglas de formación. Puede decirse, entonces, que la arqueología permite aproximarse a los discursos en tanto prácticas que forman regularmente los objetos que solo en ella tienen lugar, al tiempo que posibilitan la constitución correlativa de formas de subjetividad. En términos del epistemólogo Martin Kusch:

Lo que Foucault pretende señalar es que los objetos de un discurso (científico) no están esperando "allí afuera", sino que son más bien el resultado de un grupo de relaciones que existe dentro y entre superficies, autoridades y grillas. En otros términos, "no es suficiente abrir los ojos, prestar atención" para encontrar nuevos objetos científicos, sino que esos nuevos objetos existen solamente "bajo condicio- 
nes positivas de un grupo complejo de relaciones" (Kusch $199166)$.

Cabría destacar que este modo de problematización de los saberes en tanto prácticas discursivas permitió a Foucault plantear la oposición entre la teoría del conocimiento, que desde su perspectiva se ocuparía de dar cuenta de la relación sujeto-objeto, y la arqueología del saber que se coloca por fuera de dicha relación, dando cuenta de la formación de ambos términos en tanto inmanentes al ejercicio de la función enunciativa. Por lo tanto, consideramos que el eje de la apuesta foucaulteana radica en la problematización del saber como un modo alternativo a la elaboración de una teoría del conocimiento; puesto que no se trata, para Foucault, de problematizar los términos de la relación cognoscitiva sino de colocarse por fuera de la relación misma, dando lugar a la problemática del saber en oposición a la del conocimiento (Foucault 1994k). De lo que se trata, entonces, es de dirigir el análisis al substrato arqueológico de la constitución de las formas de subjetividad y de los objetos, para indagar la configuración del denominado a priori histórico. En términos del propio Foucault:

Yuxtapuestos, estos dos términos hacen un efecto un poco resonante; entiendo designar con ello un a priori que no sería condición de validez para unos juicios, sino condición de realidad para unos enunciados. No se trata de encontrar aquello que podría hacer legítima una aserción, sino de aislar las condiciones de emergencia de unos enunciados, la ley de su coexistencia con otros, la forma específica de su modo de ser, los principios según los cuales ellos subsisten, se transforman y desaparecen (Foucault 1969 167).

La arqueología del saber se caracteriza, entonces, por llevar a cabo un trabajo archivístico sobre los discursos que, al abordarlos en tanto prácticas, permite dar cuenta de sus condiciones de posibilidad, al tratar los documentos como monumentos. En términos de la especialista Maria Muhle:

La puesta en cuestión del documento realizada por la arqueología foucaulteana se dirige, por lo tanto, en contra de 
un modelo de historia que aspira al reconocimiento de un pasado inscripto en los documentos y luego desvanecido. El documento no es más para la historia el rastro de las cosas pasadas, "la materia inerte de la historia", por el contrario, es en el "tejido documentario" mismo que la historia busca definir las unidades, los conjuntos, las series, las relaciones (Muhle 2012 190).

En torno a lo cual cabe destacar que, en la medida en que la arqueología se despliega por medio del abordaje de la historia efectiva de las prácticas discursivas, la misma se ubica en una posición de exterioridad respecto de los discursos de cuya formación se ocupa. En ese sentido, permite llevar a cabo una indagación que no se encuentra motorizada por preocupaciones normativas y que se realiza por medio del trabajo de archivo que prescinde de tomar la ciencia actual como vara para la realización de una historia en clave teleológica. Como lo sostuviera en el prefacio de su trabajo precedente, Les mots et les choses, de 1966:

No se interrogarán los conocimientos descritos en su progreso hacia una objetividad en la que la ciencia actual pudiera reconocerse; lo que se pondrá en juego, es el campo epistemológico, la episteme en que los conocimientos, vistos más allá de todo criterio referente a su valor racional o a sus formas objetivas, hunden su positividad y manifiestan así una historia que no es la de su perfección creciente, sino más bien la de sus condiciones de posibilidad (...). Más que de una historia en el sentido tradicional del término, se trata de una "arqueología" (Foucault 1966 13).

Teniendo presentes las distintas referencias a la producción arqueológica foucaulteana que hemos introducido a lo largo de este apartado, debemos revisar la manera en que Foucault contrapone la indagación arqueológica respecto de una reflexión epistemológica de carácter normativo en el último capítulo de L'archéologie du savoir:

En este nivel la cientificidad no sirve de norma: lo que se busca dejar al desnudo, en esta historia arqueológica, son las prácticas discursivas en la medida en que dan lugar a 
un saber, y ese saber toma el estatuto y el rol de ciencia. (...) En el enigma del discurso científico, lo que pone en juego no es el derecho a ser una ciencia, es el hecho de que existe (Foucault 1969 249-251).

Buscando desentrañar el discurso foucaulteano en su especificidad, consideramos que la arqueología del saber no pretende configurarse como una teoría del conocimiento alternativa, que problematizaría la relación sujeto-objeto, sino que entre sus objetivos se destaca el dar cuenta de los modos históricos de constitución de ambos términos al remitirlos a sus condiciones de posibilidad. Por lo tanto, en el marco de la distinción entre saber y conocimiento, que habilita el desanclaje de la epistemología de las ciencias humanas del interior de la relación sujeto-objeto, la perspectiva arqueológica permite elaborar un registro de problematización que prescinde de tomar la ciencia actual historiográficamente en términos teleológicos y epistemológicamente de modo normativo. En ese sentido, citamos nuevamente el trabajo de Edgardo Castro, quien sintéticamente planteó, en relación a la historia arqueológica, que:

Este tipo de análisis prescinde tanto de la ciencia actual como norma del trabajo histórico cuanto de la formalización como criterio de investigación. Ella procura establecer cómo se ha formado un saber determinado, es decir, cómo se ha constituido un dominio de objetos, cómo se han definido las posiciones subjetivas, cómo ha surgido un conjunto de conceptos y cómo funciona, cómo se relaciona este nuevo dominio del saber con otros, etcétera (Castro 1995 218- 219).

La problematización del saber permite dar cuenta del modo en que históricamente -de manera inmanente a cierta disposición epistémicase constituyen objetos y posiciones de sujeto. Por lo tanto, en lugar de brindar herramientas para la realización de una crítica normativa respecto de la "objetividad cognoscitiva" de las ciencias humanas, la crítica arqueológica se desplaza hacia la problematización de las formas de objetivación. De este modo, puede decirse entonces que el trabajo epistemológico del arqueólogo "no se basta a sí mismo", sino que se 
encuentra jalonado por preocupaciones de índole ontológico-política respecto de la actualidad. Tal como podemos rastrearlo de manera recurrente en los trabajos de Foucault, por ejemplo, en sus investigaciones acerca de la constitución de la locura (Foucault 1972), el hombre (Foucault 1966), la delincuencia (Foucault 1975), la sexualidad y la anormalidad (Foucault 1976), el mercado, el homo œeconomicus y el capital humano (Foucault 2004a 2004b).

Abordaje de los saberes acerca de "lo humano" que, en lugar de cuestionar su "falta de objetividad" y contraponer un "modo adecuando de conocer", se ejercita por medio de la problematización acerca de la formación de dichos objetos, desplegando así un conjunto de herramientas para la crítica epistemológica de las ciencias humanas que se encuentra jalonada por la preocupación ontológico-política respecto de la constitución de la actualidad. Así, en lugar de "la denuncia" del carácter ideológico, sesgado o pseudocientífico de dichos saberes, encontramos en la crítica foucaulteana una indagación acerca del modo en que la formación de dichas ciencias contribuye a la constitución de focos de experiencia, articulándose con el ejercicio del poder por medio de prácticas como el examen y la disciplina (Foucault 1975), la regulación biopolítica (Foucault 1976) y las formas de gobierno económico (Foucault, 2004a 2004b).

\section{A modo de cierre}

"Hacer la historia de la objetivación, no explicar lo que se ha pensado acerca de la locura en la modernidad, no cómo las sociedades occiden-

tales se han comportado con respecto a ella, sino cómo se ha constituido un objeto de saber y de práctica social y política que tenía los caracteres de la locura; por lo tanto, hacer la historia de la constitución de los objetos significaba, para Foucault, contribuir a la problematización del presente (...)" (Giordano 2007 70).

A partir de lo descrito en los parágrafos precedentes, sostenemos que la arqueología foucaulteana de los saberes acerca de "lo humano", en lugar de contribuir a la realización de la epistemología normativa, permite llevar a cabo una crítica ontológico-política respecto de la 
actualidad, interpretación que erigimos a partir de la puesta en juego de tres pares categoriales, dos introducidos por el propio Foucault y el tercero incorporado por quien escribe estas líneas. La articulación de los pares introducidos por Foucault permitió perfilar dos senderos para el trabajo filosófico que, declinado hacia la epistemología de las ciencias humanas, daría lugar a un tercer par. Tenemos, por un lado, entonces, una filosofía analítica de la verdad en general, preocupada por las posibilidades del conocimiento y sus límites infranqueables, es decir, por la elaboración de una teoría del conocimiento que, respecto de las ciencias humanas, permitiría perfilar una crítica epistemológica normativa acerca de su objetividad cognoscitiva. Frente a lo cual, la propuesta foucaulteana de llevar a cabo una ontología de la actualidad se entronca con el abordaje arqueológico del saber y da lugar, respecto de las ciencias humanas, a una crítica epistemológica que, en lugar de bastarse a sí misma, se configura como un aporte a la problematización ontológico-política de la actualidad, por medio de la crítica de las formas de objetivación.

Puede decirse, entonces, que nos encontramos frente a dos inflexiones posibles para la crítica epistemológica de las ciencias humanas, el camino de la "analítica de la verdad" y la teoría del conocimiento, que da lugar al "perenne problema" de la "objetividad cognoscitiva" de dichas ciencias; inflexión frente a la que el discurso foucaulteano introduce un desplazamiento del eje de problematización, perfilando una crítica epistemológica y ontológico-política de las formas de objetivación inmanentes a la formación de los saberes. Así, en lugar de cuestionar epistemológicamente el discurso de las mencionadas ciencias en función de su supuesta "falta de objetividad" y de oponerles una forma "más adecuada" de abordar sus objetos, de lo que se trata es de problematizar el modo en que sus formas de objetivación contribuyen a la constitución ontológico-política de la actualidad.

\section{Bibliografía}

Beresñak, Fernando. "El espacio de la verdad foucaulteana". El Banquete de los Dioses. Revista de Filosofía y Teoría Política Contemporáneas 2/2, (2014). 
Brossat, Alain. "L'archive et les archives.Archéologie des discours et gouvernement des vivants". Materiali Foucaultiani 2/4 (2013).

Castro, Edgardo. Pensar a Foucault: interrogantes filosóficos de La arqueología del saber. Buenos Aires: Editorial Biblos, 1995.

Castro, Edgardo. Lecturas foucaulteanas. Una historia conceptual de la biopolítica. Buenos Aires: UNIPE: Editorial Universitaria, 2011a.

Castro, Edgardo. Diccionario Foucault. Temas, problemas y autores. Buenos Aires, $2011 \mathrm{~b}$.

Castro-Gómez, Santiago. Historia de la gubernamentalidad. Razón de Estado, liberalismo y neoliberalismo en Michel Foucault. Bogotá: Siglo del Hombre Editores, 2010.

Defert, Daniel. "Situation du Cours", Leçons sur la volonté de savoir. Cours au Collège de France. 1970 - 1971, Michel Foucault. Paris: Éditions Gallimard SEUIL, 2011.

Fontana, Alessandro, Bertani, Mauro. "Situation du Cours", II faut défendre la société. Cours au Collège de France, 1976, Michel Foucault. Paris: Éditions Gallimard SEUIL, 1997.

Foucault, Michel. Naissance de la clinique. Une archéologie du regard médical. Paris: Presses Universitaires de France, 1963.

Foucault, Michel. Les mots et les choses. Une archéologie des sciences humaines. Paris: Gallimard, 1966.

Foucault, Michel. L'archéologie du savoir. Paris: Éditions Gallimard, 1969.

Foucault, Michel. Histoire de la folie à l’âge classique. Deuxième édition, Paris: Éditions Gallimard, 1972.

Foucault, Michel. Surveiller et punir. Naissance de la prison. Paris: Éditions Gallimard, 1975.

Foucault, Michel. Histoire de la sexualité 1. La volonté de savoir. Paris: Éditions Gallimard, 1976.

Foucault, Michel. «Qu`est-ce que la Critique? (Critique et Aufklrärung)». Bulletin de la Société française de Philosophie, Paris, 84/2 (1990).

Foucault, Michel. "Entretien avec Michel Foucault", Dits et écrits. 1954-1988. IV. 1980 - 1988, Michel Foucault. Paris: Éditions Gallimard, 1994a.

Foucault, Michel. "Qu`est-ce qu`un auteur ?", Dits et écrits. 1954-1988. I.1954 - 1969, Michel Foucault. Paris: Éditions Gallimard, 1994b. 
Foucault, Michel. "Qu`est-ce que les Lumières ?", Dits et écrits. 1954-1988. IV. 1980 - 1988, Michel Foucault. Paris, Éditions Gallimard, 1994c.

Foucault, Michel. "Qui étés-vous, professeur Foucault?", Dits et écrits. 19541988. I. 1954-1969, Michel Foucault. Paris: Éditions Gallimard, 1994d.

Foucault, Michel. "La philosophie structuraliste permet de diagnostiquer ce qu'est 'aujourd'hui'"', Dits et écrits. 1954-1988. I.1954 - 1969, Michel Foucault. Paris: Éditions Gallimard, 1994e.

Foucault, Michel. "Foucault répond à Sartre", Dits et écrits. 1954-1988. I.1954 - 1969, Michel Foucault. Paris: Éditions Gallimard, 1994f.

Foucault, Michel. "Réponse à une question", Dits et écrits. 1954-1988. I.1954 - 1969, Michel Foucault. Paris: Éditions Gallimard, 1994g.

Foucault, Michel. "Qu`est-ce qu’un philosophe?", Dits et écrits. 1954-1988. I.1954 - 1969, Michel Foucault. Paris: Éditions Gallimard, 1994h.

Foucault, Michel. "Nietzsche, la généalogie, Ihistoire", Dits et écrits. 19541988. II.1970 - 1975, Michel Foucault. Paris: Éditions Gallimard, 1994.

Foucault, Michel. "La vérité et les formes juridiques", Dits et écrits. 1954-1988. II. 1970 - 1975, Michel Foucault. Paris: Éditions Gallimard, 1994j.

Foucault, Michel. "Sur l’archéologie des sciences. Réponse au Cercle d’épistémologie", Dits et écrits. 1954-1988. I.1954 - 1969, Michel Foucault. Paris: Éditions Gallimard, 1994k.

Foucault, Michel. Sécurité, Territoire, Population. Cours au Collège de France. 1977-1978. Paris: Éditions Gallimard SEUIL, 2004a.

Foucault, Michel. Naissance de la biopolitique. Cours au Collège de France. 1978-1979, Paris: Éditions Gallimard SEUIL, 2004b.

Foucault, Michel. Le gouvernement de soi et des autres. Cours au Collège de France. 1982-1983. Paris: Éditions Gallimard SEUIL, 2008.

Foucault, Michel. Leçons sur la volonté de savoir. Cours au Collège de France. 1970-1971. Paris: Éditions Gallimard SEUIL, 2011.

Giordano, Claudia. Un`idea di filosofia. Michel Foucault, dalla critica dell'antropologia all etica de la cura di sé. Napoli. Tesi di Dottorato di ricerca in Filosofia Moderna e Contemporanea - Ciclo XX - Indirizzo: Filosofia e Teoria della Scienze Umane, Istituo Italiano di Scienze Umane. Scuola di Alta Formazione nelle Scienze Umane e Sociali dell Università degli Studi di Napoli Federico II, 2007. 
Gros, Frédéric. "Situation du Cours", Le gouvernement de soi et des autres. Cours au Collège de France. 1982-1983, Michel Foucault. Paris: Éditions Gallimard SEUIL, 2008.

Gutting, Gary. Michel Foucault's archaeology of scientific reason. Cambridge: Cambridge University Press, 1989.

Han, Béatrice. L'ontologie manquée de Michel Foucault. Entre l'historique et le transcendantal. Grenoble: Éditions Jérôme Million, 1998.

Kusch, Martin. Foucault's strata and fields. An Investigation into Archaeological and Genealogical Science Studies. Boston: Springer Science + Business Media Dordrecht, 1991.

Mascaretti, Giovanni Maria. "Michel Foucault on Problematization, Parrhesia and Critique". Materiali Foucaultiani 3/5-6: (2014).

Muhle, Maria. "Histoire(s) de la vie de Canguilhem a Foucault", Epistemology and History. From Bachelard and Canguilhem to Today's History of Science, A.A.V.V. Max Planck Institute for the History of Science, 2012.

Nosetto, Luciano. Discursos y estrategias. Michel Foucault y la política. Buenos Aires, Tesis de Doctorado en Ciencias Sociales, Facultad de Ciencias Sociales, Universidad de Buenos Aires, 2010.

Paltrinieri, Luca. "Archeologia della volontà. Una preistoria delle Lezioni sulla volontà di sapere". Quadranti. Rivista Internazionale di Filosofia Contemporanea 2/1 (2014).

Raffin, Marcelo. "El pensamiento de Gilles Deleuze y Michel Foucault en cuestión: las ideas en torno del poder, el sujeto y la verdad". Lecciones y Ensayos 85 (2008).

Raffin, Marcelo. "Natureza e política: uma tensão no pensamento de Michel Foucault". Ágora Filosófica 1/2 (2014).

Raffin, Marcelo. "La verdad y las formas políticas: la lectura temprana de la tragedia de Edipo en Michel Foucault". Anacronismo e Irrupción. Revista de Teoría y Filosofía Política Clásica y Moderna 5/8 (2015).

Sabot, Philippe. Lire Les mots et les choses de Michel Foucault. Paris: Presses Universitaires de France, 2006.

Wallenstein, Sven-Olov. "Introduction: Foucault, Biopolitics, and Governmentality", Foucault, Biopolitics and Governmentality, Nilsson, Jakob, Wallenstein, Sven-Olov (Eds.). Stockholm: Södertörn, Södertörn University, The Library, 2013. 